\title{
MedienPädagogik
}

Zeitschrift für Theorie und Praxis der Medienbildung

Themenheft Nr. 42: Optimierung in der Medienpädagogik.

Forschungsperspektiven im Anschluss an den 27. Kongress der DGfE

Herausgegeben von Patrick Bettinger, Klaus Rummler und Karsten D. Wolf

\section{Subjektwissen als Bildungsperspektive junger Erwachsener}

\section{Self-Tracking-Apps als digitale Optimierungstechnologien im Spannungsgefüge von Kontrolle und Selbstermächtigung}

Bianca Burgfeld-Meise und Lukas Dehmel

\begin{abstract}
Zusammenfassung
Das Individuum muss in der Risikogesellschaft Lebensentscheidungen treffen und damit verbundene Risiken eigenverantwortlich tragen (Beck 1986). Zudem sind Optimierungsimperative allgegenwärtig, die als Disziplinierungen gesellschaftlich produktiv und für das Individuum zugleich einschränkend sein können (Foucault 1976, 265). Durch technischen Fortschritt gleichen diese heute mehr denn je «ultra-schnellen Kontrollformen mit freiheitlichem Aussehen» (Deleuze 1993, 255). Besonders sichtbar werden sie als Self-TrackingApps. Sie versprechen z. B. eine Steigerung der körperlichen Fitness oder der individuellen Leistungsfähigkeit. Jedoch sind etwa durch Datenmissbrauch, utopische Zielvorstellungen und Verdatung auch zahlreiche Risiken mit der Nutzung verbunden (Selke 2016). In unserem Beitrag werden wir die unterschiedlichen Facetten des Phänomens auf Basis einer qualitativen Studie mit Studierenden diskutieren. Wir arbeiten in einer zweiteiligen Analyse heraus, wie sich verändernde Selbst-/Weltbezüge im Spannungsfeld zwischen Kontrolle und Selbstermächtigung konstituieren. Zur Verdeutlichung rekonstruieren wir an einem Beispiel, wie sich das konturierte Spannungsfeld in die Self-Tracking-App Time Reduction einschreibt, und zeigen an Interviewmaterialien, wie sich ein Student zu diesem in Beziehung setzt.
\end{abstract}

Subject knowledge as an educational perspective of young adults. Self-tracking-apps as digital optimization technologies between surveillance and self-empowerment

\begin{abstract}
In risk societies individuals are responsible for their own life decisions (Beck 1986). Furthermore they are confronted with optimization imperatives and their productive and restrictive influences (Foucault 1976, 265). Digital media and data analytics are new forms of discipline technologies in this perspective. They seem to appear like a free choice but they contain surveillance structures that are internalized by individuals (Deleuze 1993).
\end{abstract}


Especially self-tracking-apps illustrate this point in particular. They promise a growing health or capability. But on the other hand they contain different risks like data abuse, misguided ideals, and datacizing (Selke 2016). In this regard our paper discusses different aspects of this phenomenon based on a qualitative study with university students. In a two-step analytical approach we investigate the subject related changes between surveillance and self-empowerment in perspective of education theory. As an example we present an explorative investigation of the app Time Reduction and its surveillance structures. In a second step we explore its interaction with a university student and his following subject related changes.

\section{1. $\quad$ Einleitung}

Es gibt wohl kaum Technologien, die so unmittelbar auf Optimierung ausgelegt sind, wie Self-Tracking-Apps. Diese digitale Selbstbeobachtung ist beliebt: Sei dies nun der Schrittzähler beziehungsweise die Laufstreckenmessung in Fitness-Apps oder die Dokumentation der Nahrungsaufnahme, der geographischen Daten (Urlaube, Besuche, Wanderungen etc.) und vieles mehr. Datenschutz und Kontrolle stehen grundsätzlich mit diesen Onlinediskursen und insbesondere bei Self-Tracking-Diensten im Fokus und werden nicht selten kritisch betrachtet. Wir werden den Diskurs um Datenschutz und Kontrolle daher auf verschiedenen Eben betrachten. Dazu zählen entsprechend Perspektiven der protokollierenden medialen Dienste und der Datenverwertung durch Drittanbieter, aber auch das Verhältnis des kontrollierten Individuums zum Medium und zu sich selbst (Foucault 1976, Deleuze 1993). Dabei möchten wir insbesondere auch die Ermächtigung als Gegenstand des Diskurses in den Vordergrund rücken. Unser Hauptaugenmerk bezieht sich somit auf eine prozessuale Verbindung, innerhalb derer sowohl Menschen, aber auch die über das Smartphone aufgerufene App miteinander in soziomedialen Austausch treten. Der Beitrag setzt sich in diesem Zusammenhang mit der Frage auseinander, wie diese Verwicklung von Mensch und Self-Tracking-App zwischen Kontrolle und Selbstermächtigung der Individuen hervorgebracht wird. Wir werden unsere Herangehensweise zuerst theoretisch aufarbeiten und folgend anhand einer empirischen Analyse reflektieren.

Dafür widmen wir uns zunächst den theoretischen Perspektiven von Foucault und Deleuze auf Disziplinierung und Kontrolle (Foucault 1976, Deleuze 1993) ${ }^{1}$ (2.) und beschäftigen uns anschliessend für unsere Analyse mit dem leitenden Ordnungsverhältnis von Mensch und Self-Tracking-App (3.). Für die empirische Reflexion

1 Foucault und Deleuze werden als Heuristiken zur Analyse des empirischen Materials gelesen. Dabei geht es nicht um die Operationalisierung der Theorien sondern vielmehr darum, die Grundgedanken der Theorien zu fassen und mit diesen die aktuellen Phänomene der Optimierung im Sinne von Disziplinierung und Kontrolle zu betrachten. Sie sind also eher eine Hilfestellung, um neue Phänomene einordnen zu können. Die Theorien können, als Heuristiken betrachtet, auch bei ihrer Nichtanwendbarkeit zu neuen Erkenntnissen führen. 
nutzen wir qualitative Datenmaterialien aus einem Forschungsseminar zu Lifelogging-Praktiken (4.). Um den zuvor umrissenen Ansatz aufzugreifen, setzen wir eine Analyse in zwei Schritten um. Zuerst untersuchen wir die Self-Tracking-App als medial-materiellen Aktanten, im Anschluss rücken wir die Seite des menschlichen Akteurs in den Mittelpunkt und interpretieren die im Seminar erhobenen Interviewtranskripte (5). Danach setzen wir beide Analysen in Zusammenhang und kommen so zu einem abschliessenden Fazit hinsichtlich der Konstruktionsweisen von Erfahrungen zwischen Kontrolle und Selbstermächtigung für den Hybrid-Akteur ${ }^{2}$ insgesamt. Im Fazit (6.) unterziehen wir die Ergebnisse unserer Interpretation schliesslich einer bildungstheoretischen Reflexion, indem das unterschiedlich entstehende Subjektwissen in diese Kontexte eingerückt wird (Burgfeld-Meise 2020). Subjektwissen entsteht also erst in der Analyse. Im Rahmen unserer Studie sind das subjektive Überzeugungen des Subjekts, subjektiv generierte empirische Daten und die (auch unbemerkte) Datensammlung über das Subjekt durch Dritte.

\section{Optimierung zwischen Kontrolle und Selbstermächtigung}

Self-Tracking-Apps können auf unterschiedliche Arten als Überwachungsinstrumente interpretiert werden. Kontrolle und Ermächtigung liegen dabei gar nicht so weit voneinander entfernt, wie man zunächst vermuten könnte. Tatsächlich kann Kontrolle auch ein Element der Selbstermächtigung sein. Im Folgenden werden wir zuerst aus theoretischer Perspektive aufzeigen, wie fliessend und vielschichtig diese Prozesse sein können.

Um das Phänomen der Überwachung zu konturieren, ist ein Blick auf Michel Foucaults (1976, 251-93) Ausführungen sinnvoll. Foucault betrachtet in dieser Schrift über grössere Zeiträume hinweg die Massnahmen von Machtausübungen zum Überwachen und Strafen und leitet daraus gesellschaftliche Funktionen ab. ${ }^{3}$ In früheren Gesellschaften, den sogenannten Souveränitätsgesellschaften, oblag es dem oder der souverän Herrschenden, über Leben und Tod von Delinquentinnen und Delinquenten zu bestimmen. Nicht selten wurde in diesem Zuge der Körper des Delinquenten oder der Delinquentin versehrt oder Straftäterinnen und Straftäter in dunkle Verliese gesperrt, in denen sie bei langen Haftstrafen starben. Ähnlich verhielt es sich beim Ausbruch der Lepra: Kranke wurden nicht behandelt, sondern vor den Toren der Stadt sich selbst und somit dem Tode überlassen. Mit dem Ausbrechen der Pest fand, Foucault zufolge, eine Veränderung statt: Es wurden umfassende Massnahmen zur Separation von kranken und gesunden Menschen ergriffen. Damit einhergehend wurden Dokumentationen über den Gesundheitsstatus angelegt. Ein

2 Der Begriff wird in Abschnitt 3 näher erörtert.

3 Foucault stellt dabei jedoch nicht die Existenz des Subjekts in Abrede. Vielmehr sensibilisiert er mit seinen Theorien dafür, dass das Subjekt nicht allmächtig ist, sondern, in Machtstrukturen eingefasst, jeweils spezifische Subjektivitäten entwirft (zur Rolle des Subjekts bei Focucault siehe weiterführend Bublitz 2014). 
strenges Regularium wurde durchgesetzt, um die Durchmischung der Menschen zu verhindern und damit die Verbreitung der Pest zu verhindern. Ereignete sich ein Todesfall, wurden entsprechende Routinen durchgesetzt, um die Verbreitung weiterer Infektionen zu unterbrechen. Foucault interpretiert diese Entwicklung als Disziplinierungsmassnahmen, die tief in den individuellen und gesellschaftlichen Körper eindringen und ihn somit disziplinieren.

Besonders anschaulich stellt er die Disziplinierungsmacht mit Benthams Modellentwurf des Panopticons als Perfektion eines disziplinierenden Gefängnisses dar (ebd., 256-63). Das Panopticon ist kreisförmig angelegt, in der Mitte befindet sich der erhöhte, aber nicht einsehbare Turm der Wärterin oder des Wärters. Die Zellen der Insassinnen oder Insassen sind um den Turm herum kreisförmig angeordnet, sind von vorn und hinten lichtdurchflutet aber zu den Seiten hin durch Mauern getrennt. So sind die Insassinnen und Insassen permanent sichtbar, können jedoch ihrerseits nichts einsehen. Dies unterstreicht ein in der Architektur eingelassenes asymmetrisches Machtverhältnis, welches sich selbst stabilisiert. Dadurch, dass die Insassinnen und Insassen immer beobachtet werden können, aber nie wissen, ob sie beobachtet werden (da der Turm der Wärterinnen oder Wärter uneinsehbar ist), disziplinieren sich die Insassinnen und Insassen zunehmend selbst. Das Machtverhältnis wird internalisiert. Zudem ist es nicht wichtig, dass die Wärterinnen und Wärter beobachten, dies könnten auch andere Menschen tun, um das Machtverhältnis zu erhalten (ebd.).

Gilles Deleuze entwickelt den Ansatz Foucaults weiter. Er sieht die disziplinierenden Institutionen in einer Krise, was sich in stetig notwendigen Reformen manifestiert. Damit einhergehend müssen die sogenannten Einschliessungsmilieus (bspw. Gefängnisse, Krankenhäuser, aber auch Schulen) der Disziplinargesellschaften neue Formen entwickeln. Ein Phänomen dieser Entwicklungen sind die «ultra-schnellen Kontrollformen mit freiheitlichem Aussehen» (Deleuze 1993, 255). Beispiele hierfür sind etwa elektronische Fussfesseln als neue Form des Strafvollzugs oder aber, weniger dramatisch, Telematiktarife in der Versicherungsbranche. Die Kontrollen sind dabei weniger körperlich als bei Foucault gedacht, Deleuze benutzt den Begriff «Chiffre» (ebd., 258), um dies zu akzentuieren. Obwohl Deleuzes Ausführungen im Original von 1993 sind, lassen sich diese Mechanismen am besten anhand von Datensammlung, Tracking und Big Data Analytics verdeutlichen. Daten werden nahezu zu jedem Zeitpunkt durch Tracking von Handydaten, Navigationssystemen, Kartenzahlungen, Internetaktivitäten und dergleichen mehr gesammelt. Diese Daten können wiederum an Dritte verkauft werden und damit Ausgangspunkt umfassender digitaler Profile sein, die später den Zugang oder aber den Ausschluss von Dienstleistungen, Waren oder Rechten nach sich ziehen können - die Schufa wäre dafür wohl ein prominentes Beispiel. Zudem ergibt sich das Problem der Verdatung (Selke 2016, Weich 2017). Immer mehr Bereiche der individuellen Lebensführung können durch Self-TrackingApps in Kennzahlen operationalisiert und in ein Bewertungssystem integriert werden, das nicht selten stetige Optimierung verfolgt. 
Dadurch ergeben sich gleich mehrere Problemfelder: Zunächst werden Dinge der individuellen Lebensführung pathologisiert und durch Kennzahlen, deren tatsächliche Aussagekraft für die Nutzenden schwer nachzuvollziehen sind, vermeintlich objektiviert (Dehmel und Burgfeld-Meise 2020). Schliesslich sind damit stetige ideale Optimierungen verbunden. Veranschaulichen lässt sich dies am Beispiel der Schlaf-Tracking-Apps: Zunächst einmal ist Schlaf durch die Apps und dazugehörige Messinstrumente ein krisenhaftes Unterfangen. Durch die Operationalisierung in unterschiedlichste Kennzahlen ist Schlaf nicht nur ausreichend oder nicht ausreichend, gut oder schlecht, sondern genau auf die Sekunde, nach vermeintlichen unterschiedlichen Schlaf- und Traumphasen, Atmungsprotokollen, Pulsfrequenzen etc. berechenbar (evtl. mit anderen Kennzahlen triangulierbar) und unterliegt somit ganz vieler potenzieller Störungsquellen. Das Subjekt generiert so technologiegestützt neues vorher unzugängliches Wissen über sich selbst. Zudem ergibt sich dadurch ein Bild, das eine funktionalisitische Perspektive auf das Individuum und dessen Körper unterstützt. Durch die Vielzahl unterschiedlicher Self-Tracking-Technologien und stetig wachsender Einsatzbereiche können umfassende gesellschaftliche Optimierungsimperative entstehen. Zudem basieren Self-Tracking-Apps nicht zwingend auf wissenschaftlichen Erkenntnissen. Die Erhebung und Aussagekraft der Messdaten ist ebenso nicht selten problematisch.

Es wäre jedoch zu eindimensional, unsere Thematik nur auf diese problematischen Aspekte hin zu denken. Auch Foucault betont, dass Disziplinierung natürlich einerseits negative Implikationen für das Individuum haben kann, aber anderseits auch dazu in der Lage ist, die «Gesellschaftskräfte zu steigern» (Foucault 1976, 267). Foucault meint damit in seinen Beispielen, Delinquentinnen und Delinquenten nach der Haftstrafe wieder produktiv in die Gesellschaft einzugliedern, Kranke zu heilen, Kinder zu bilden etc. Übertragen auf unser Beispiel können Kontrollelemente für das Individuum unter risikogesellschaftlichen Lebensbedingungen eine Ermächtigungsstrategie sein. Wie Beck (1986, 205-19) in seinen Ausführungen verdeutlicht, sieht sich das Individuum in der fortschreitenden Moderne mit der Erosion traditioneller Gewissheiten konfrontiert. Dies kann sich im Verlust tradierter Erwerbslebensläufe, Religion, aber auch wissenschaftlicher Erkenntnisse zeigen. Trotz dieser Bedingungen wird das Individuum viel stärker als aktive Konstrukteurin oder aktiver Konstrukteur der eigenen Lebensführung gesehen, die oder der gleichzeitig eigene Entscheidungen treffen muss, obwohl die Konsequenzen dieser Entscheidungen nicht absehbar sind (ebd.). Es ist also gar nicht leicht für das Individuum, Orientierung zu finden und entscheidende Weichenstellungen vorzunehmen. Unter diesen Bedingungen können digitale Tracking-Technologien eine gewisse Last der Entscheidung abnehmen oder über Normwerte eine gewisse Orientierung anbieten (Selke 2016, 314-15). Nicht zuletzt ist es eine ebensolche Entlastung, wenn ein Teil der Verantwortung an die Tracking-Technologien abgeben werden kann. 
Wie argumentiert wurde, liegen Ermächtigung, Kontrolle und Optimierung ganz nah beieinander und können sich, je nach Perspektive, überlagern oder die Vorzeichen wechseln. Wenn das Subjekt bspw. über eine Allergie-App Nahrungsaufnahme und Umwelteinflüsse protokollieren kann, dürfte solches sehr hilfreich sein, eine unbekannte Allergie zu identifizieren und Kontrolle über das körperliche Wohlbefinden zurückzubekommen. Dies ist auch eine Ermächtigungsstrategie, da das Individuum aktiv seine Lage verbessert. Gleichzeitig können im Hintergrund App-Hersteller ganz andere Interessen verfolgen. Ihre Motive und Strategien sind, ganz ähnlich wie im panoptischen Turm, nicht ohne weiteres einsehbar. Es kann sein, dass jenseits der erhobenen Daten auch Zugang zu allen weiteren Handydaten erlangt wird, umfassende Metadaten gespeichert, Ernährungs- und Freizeitgewohnheiten gesammelt und ausgewertet und wiederum an Dritte gewinnbringend verkauft werden.

Die Self-Tracking-Apps sind insgesamt betrachtet häufig im Konnex von Kontrolle und Optimierung zu verorten. Es geht darum, die Gesundheit zu stärken, Gewicht zu reduzieren, mehr Sport zu treiben, die Beliebtheit zu erhöhen, mehr Wasser zu trinken, besser zu schlafen und dergleichen mehr. Gerade um das Self-Tracking von Sport, Diäten, Muskelaufbau und dessen fotografische Reproduktion entfaltet sich ein umfassendes Optimierungsimperativ, in welchem konträre Haltungen dann eher als Desinteresse an Gesundheitsthemen stigmatisiert werden können.

\section{3. Überlegungen zur empirischen Analyseperspektive}

Diese Überlegungen überführen wir nun in eine explorative empirische Analyse. Eine umfassende empiriegesättigte Theorie zu entwerfen ist dabei nicht das Ziel, sondern vielmehr, anhand des Einzelfalls die im Theoriekapitel aufgeworfenen Strukturprinzipien zwischen Mensch und Self-Tracking-App empirisch verstehbar zu machen. Wir richten uns hier nach den Überlegungen einer pädagogischen Kasuistik, die in erkenntnisgenerierender Absicht gerade die Stärken sinnverstehender Fallanalysen mithilfe rekonstruktiver Sozialforschungsmethoden betont (Hummrich 2016, 29-30). Sie nimmt an, dass der Einzelfall immer der Ausdruck allgemeiner Bedingungen und Regeln ist und vice versa (Hummrich rekurriert hier auf das von Oevermann bestimmte dialektische Verhältnis von Allgemeinem und Besonderem). Das Allgemeine ist dieser Grundlegung nach nur über den empirisch greifbaren Einzelfall zugänglich, «[...] die Interpretation der besonderten Erscheinungen beinhaltet die Rekonstruktion einer besonderen Erscheinung, die aber auf der Grundlage allgemeiner Regeln erfolgt und auf diese allgemeinen Regeln zurückgeführt wird» (ebd., 29). Anhand der im Folgenden explorierenden Einzelfallanalyse werden so Aussagen über diesen spezifischen Fall möglich, die aber vor dem Hintergrund allgemeiner Strukturprinzipien gelesen werden müssen. Es lohnt also ein vertiefender empirischer Blick auf den Einzelfall. 
Das herangezogene Beispiel ist hier ein Student, der mithilfe der Self-TrackingApp «Time Reduction» ${ }^{4}$ weniger Zeit mit dem Smartphone verbringen möchte (siehe nächster Abschnitt). Wie zuvor schon angedeutet wird am Beispiel dieses Falls die prozessuale Verbindung zwischen Mensch und App akzentuiert. Beide treten in ein sozio-mediales Austauschverhältnis, in das Kontroll- und Selbstermächtigungspraktiken eingelagert sind. Wie beispielsweise schon von Bettinger (2017) vorgeschlagen wurde, nutzen wir für die empirische Reflexion dieser In-Verhältnis-Setzung einige der Überlegungen Latours und Nohls zur Akteur-Netzwerk-Theorie (ANT), die sich bereits in einem vorangegangenen Beitrag als forschungsheuristischer Rahmen für Analysen zwischen Menschen und Self-Tracking-Apps als äusserst produktiv erwiesen haben (Dehmel und Burgfeld-Meise 2020). Diese zuvor entfalteten Perspektiven (ebd., 40-41) ${ }^{5}$ werden in diesem Beitrag daher aufgegriffen und für die folgende Untersuchung nutzbar gemacht.

Menschliche Handlungen - davon ist die ANT überzeugt - werden nicht durch materielle (vor allem auch technische) Dinge festgelegt, die Menschen können aber genauso wenig vollends über die Dinge verfügen (Latour 2006, 484-99). Um die Relation zwischen ihnen zu bestimmen, wählt Latour einen Mittelweg. Menschen und Dinge agieren in soziomateriell ausgehandelten gemeinschaftlichen Handlungskontexten, treten in Interaktion miteinander und vereinigen sich zu zusammenhängenden «Hybrid-Akteure[n]» (ebd., 488). Beide sind also zentral wichtig für sozialwissenschaftliche Untersuchungen, es sind immer die Menschen gemeinsam mit den Dingen, die eine Praxis so hervorbringen, wie sie ist, und nicht die Menschen oder die Dinge für sich allein (ebd., 484-99).

Den Begriff des Hybrid-Akteurs führt auch Nohl in seine pädagogischen Betrachtungen der ANT ein (Nohl 2011, 36), der Begriff spielt also ebenso für die erziehungswissenschaftliche Forschung eine zentrale Rolle. Nohl schreibt es dem dinglichen Part dieser ausgehandelten Verknüpfung zu, durch seine materiellen Merkmale einen Rahmen von potentiellen Alternativen aufzuspannen, in dem die Gebrauchsvarianten für den menschlichen Akteur oder die menschliche Akteurin oszillieren und sich weiterentwickeln (ebd., 34-40 u. 91-96). Dieser von der Self-Tracking-App im Austausch mit dem Menschen aufgespannte Rahmen überschreitet die materiell-haptischen Gesichtspunkte des Smartphones in dieser theoretischen Perspektive natürlich bei weitem. Die über den Touchscreen des Geräts medial bereitgestellten Handlungsmöglichkeiten des App-Interface sind für das gemeinschaftliche Handeln des Hybrid-Akteurs weitaus entscheidender und mitunter um einiges komplexer. Dass auch Nohl dies in seinen Betrachtungen mitdenkt, zeigt sich in seiner exemplarischen

4 Der Name der App ist anonymisiert.

5 Siehe auch die empirische Nutzbarmachung der Ausführungen Latours und Nohls zur ANT für die Erforschung des Smartphones als Akteur in der berufsvorbereitenden Bildung (Dehmel und Zick 2020, 18284) und für die Erforschung der Auseinandersetzung von Lehrkräften mit Tablets (Gerhardts, Dehmel, und Meister 2021, 135-37). 
empirischen Nutzbarmachung seiner Vorüberlegungen am Beispiel des Hybrid-Akteurs aus einer Seniorin und einer von ihr im Austausch mit einem Computer erstellten Internetseite (ebd., 102-24). Hier stehen also genauso diese medial bereitgestellten Handlungsmöglichkeiten im Vordergrund. Auch im Falle der Self-Tracking-App sind sie für die folgende empirische Analyse deutlich interessanter, trotzdem sollte die materielle Konstitution des Smartphones in diesem Kontext nicht unterschätzt werden (Dehmel und Zick 2020, 182). Aus dem Blickwinkel dieses Textes ist es folglich diese «medial-materielle Beschaffenheit» (ebd., 183) der über den Touchscreen des Smartphones dargebotenen Self-Tracking-App, die den Möglichkeitsrahmen innerhalb des gemeinsamen Handelns des Hybrid-Akteures vorstrukturiert. Die App diktiert für die gegenseitige Interaktion, welche Handlungsdurchführungen auf der menschlichen Seite zu erlernen und zu verrichten sind (ebd., 183-84; Bettinger 2017, 11-13).

Dennoch schreibt Nohl es dem Austausch mit dem Menschen zu, die Eigenheiten der Dinge in der gemeinsamen Praxis zum Tragen zu bringen und sie mitunter auch abzuändern (Nohl 2011, 40-45 u. 96). Nohl nimmt in diesem thematischen Kontext Bezug auf die Betrachtungen Rammerts und Schulz-Schaeffers (2002), die die Grundlegungen der ANT einer kritischen Prüfung unterziehen. Darauf aufbauend geht er in seinen Ausführungen davon aus, dass lediglich die menschliche Akteursseite - in unserem Fall also der Student - zum intentionalen Handeln in der Lage ist (Nohl 2011, 40-45). Den Dingen schreibt Nohl diese Fertigkeit nicht zu und meint damit explizit auch «intelligente Technologien» (ebd., 43 u. 45) - in unserem Fall also die Self-Tracking-App. Für seinen theoretischen Standpunkt zur ANT markiert dies den entscheidenden Unterschied zwischen Menschen und Dingen, was zur wichtigen Grundlage seiner weiteren Argumentation wird. Nur die Menschen können ihre routinierten Alltagspraktiken kritisch überdenken und situationssensibel anpassen, wenn es nötig wird (ebd., 40-45). Diese von Nohl prominent gesetzte Überlegung übernehmen wir und legen sie der weiteren Untersuchung zugrunde.

Aus dieser theoretischen Perspektive heraus ist es die Seltracking-App, die den Studenten auf vielfältige Weise überwacht und Handlungsoptionen vorgibt und somit gleichsam Potentiale zur Selbstermächtigung schafft. Die App und ihre algorithmische Datenverarbeitung operieren für den Studenten weitgehend im Verborgenen. Sie lässt sich in der eingeschlagenen Perspektive im Anschluss an Foucault als Panoptismus interpretieren, dessen Kontrollpraktiken für den Studenten im gegenseitigen Austausch kaum einsehbar sind und dennoch hochgradig disziplinierend wirken können. Sie schlagen sich in diesem Beispiel in normativen Vorgaben hinsichtlich der aufgewendeten Zeit auf die alltägliche Smartphone-Nutzung nieder, deren Reduktion dem Studenten neue Freiheiten in seiner Alltagsgestaltung versprechen. Sie werden als Zahlenwerte aufbereitet über das App-Interface visualisiert. Es geht in dieser Perspektive um ein potentielles (Zuviel) an Nutzungszeit, das von anderen 
produktiveren Tätigkeiten ablenkt. ${ }^{6}$ Inwieweit hier zwischen natürlich ebenso möglicher produktiver Smartphone-Nutzung unterschieden wird, muss die Analyse zeigen. Erst durch die Aufbereitung der Zahlenwerte generiert der Student über seine individuelle Nutzungszeit (Subjekt-)Wissen und kann sich potentiell als Mangelwesen wahrnehmen, das einer Verhaltensmodifikation bedarf. Optimierung ist hier also nicht mit den Kontrollpraktiken der App gleichzusetzen, sondern ist vielmehr eine Reaktion auf die dargebotenen Zahlenwerte auf der menschlichen Akteursseite.

Trotzdem ist der Student aber derjenige, der die gemeinsamen Handlungen des Hybrid-Akteurs durch seine Fähigkeit zum reflexiven Denken zielgerichtet lenkt und den bereitgestellten Möglichkeitsrahmen (intentional) zur Selbstoptimierung nutzt oder eben nicht nutzt. Es ist der Student, der die Entscheidung darüber fällt, die App überhaupt zu installieren und den Austausch zuzulassen. Die von ihr dargebotenen Zahlenwerte und vorgenommenen Einordnungen in normative Vorgaben alleine sagen noch nichts aus, sie erhalten erst im Austausch mit dem Studenten durch dessen subjektive Interpretation Bedeutung für dessen Alltag. Sie fordern den Studenten zur Reflexion seines Verhaltens auf. Ob er diesen normativen Vorgaben aber tatsächlich Vertrauen schenkt und eine (von der App als Verbesserung bewertete) Verhaltensänderung im Sinne einer auferlegten Selbstoptimierung folgen lässt, ist letztlich ihm überlassen. Es steht ihm genauso frei, die App wieder zu löschen und die Verbindung des Hybrid-Akteurs aufzulösen. Im Sinne der aufgegriffenen Perspektive Becks zur Risikogesellschaft (siehe Abschnitt 2) liegt es also auch am Studenten, die App bzw. die von ihr ausgegebenen Zahlenwerte als sinnstiftendes Element zur Orientierung in den Alltag zu integrieren - oder eben auch nicht.

\section{Datengrundlage und Forschungsmethodik}

Für die empirische Analyse greift dieser Beitrag auf Datenmaterialien aus einem Forschungsseminar mit Studierenden im jungen Erwachsenenalter zurück, die sich mit der bildungstheoretischen Reflexion von Lifelogging-Praktiken auseinandergesetzt haben ${ }^{7}$. Die Studierenden führten dazu in Kleingruppen einen Selbstversuch durch. Sie erhielten den Auftrag, sich einen zentralen Bereich ihrer Alltagsroutinen zu überlegen, mit Blick auf diese Routinen ein Optimierungsziel festzusetzen und für einen Monat ein Protokoll über das eigene Handeln zu führen. Die Festlegung des Ziels und auch die Methodik zur Umsetzung dieser Selbstprotokollierung waren gänzlich den Studierenden überlassen. Zudem konnte der Selbstversuch jeder Zeit abgebrochen werden. Nach einem Monat endete das Experiment und die Studierenden führten untereinander leitfadengestützte Interviews (Keuneke 2005) zu ihren Erlebnissen. Sie befragten sich gegenseitig und transkribierten die entstandenen Interviews anschliessend (siehe bereits Dehmel und Burgfeld-Meise 2020, 41f.).

6 Dies ist die normative Setzung der App und deren Hersteller.

7 Ein schriftliches Einverständnis zur wissenschaftlichen Weiternutzung der Daten liegt selbstverständlich vor. 
Unsere darauf aufbauende Forschung konzentriert sich auf die Transkripte, in denen während des Selbstversuchs die fokussierten Self-Tracking-Apps genutzt wurden. Im Folgenden bezieht sich der Text auf den Fall eines Studenten, der sich das Ziel gesetzt hatte, weniger oft auf sein Smartphone zu schauen und die Nutzungszeit zu reduzieren, um «weniger Zeit zu verschwenden». Dafür nutzte er die App «Time Reduction», die die Bildschirmzeit und die Entsperrungen des Smartphones protokolliert. Anders als in anderen Fällen des Samples, die sich mit der täglichen Wasserzufuhr, der Schlafzeit oder der Vermessung individueller Gefühlszustände (MoodTracking) beschäftigen, erfolgt die Verhaltensprotokollierung in diesem Fall automatisiert durch die App und ist nicht auf explizite Eingaben von Werten durch den Menschen angewiesen. Diese autonome Beobachtung des Studenten durch die App innerhalb des Hybrid-Akteurs erschien uns für die auf den Panoptismus von Foucault rekurrierende Perspektive dieses Beitrags besonders geeignet, um diesen Fall für die exemplarische Analyse heranzuziehen. Zudem ist die hierbei angelegte Optimierungslogik ausserordentlich interessant. Anders, als es für Self-Tracking-Praktiken oftmals üblich ist (z. B. Selke 2016, 312-14), liegt der Fokus hier nicht auf einem Mehr eines als erstrebenswert kodifizierten Verhaltens, um hinsichtlich der individualisierten Lebensführung bessere Resultate in einem Lebensbereich zu erzielen. Ganz im Gegenteil wird hier ein Weniger eines als schlecht bewerteten Verhaltens ins Zentrum gerückt, um sich auf andere Alltagsbereiche (etwa Lernen oder auch soziale Interaktion mit anderen) produktiver konzentrieren zu können.

Um dem zuvor skizzierten Rahmen der ANT in der empirischen Analyse Rechnung zu tragen, greifen wir die Überlegung auf, sowohl die App als medial-materiellen Akteur als auch das Interview mit dem Studenten als menschlichem Akteur zu untersuchen und so der in diesem Beitrag fokussierten Verbindung zwischen beiden zu einem Hybrid-Akteur angemessen zu begegnen (Bettinger 2017; Dehmel und Burgfeld-Meise 2020). Im Sinne der eingangs formulierten Fragestellung nach der Verwicklung von Mensch und Self-Tracking-App zwischen Kontrolle und Selbstermächtigung geht es im Sinne der aufgegriffenen Ideen der pädagogischen Kasuistik um eine sinnverstehende Analyse, die auf die Rekonstruktion von Strukturprinzipien der Hervorbringung dieses Verhältnisses anhand des besonderen Einzelfalls abzielt (siehe Abschnitt 3).

Die folgende Untersuchung interpretiert daher zunächst die während des Selbstversuchs relevant gewordene Self-Tracking-App «Time Reduction» als qualitatives Datenmaterial, das wir mit einem «App Walk Through» (Light, Burgess, und Duguay 2018) beleuchten. Hierbei steht die Frage im Mittelpunkt, wie der Möglichkeitsrahmen der App konzipiert ist und welche Handlungsalternativen er für das gemeinsame Optimierungshandeln des Hybrid-Akteurs zwischen Kontrolle und Selbstermächtigung ermöglicht bzw. ausschliesst. Im zweiten Analyseschritt rekonstruieren wir 
das transkribierte Interview mit der Objektiven Hermeneutik als etablierter ${ }^{8}$ sinnverstehender Methode rekonstruktiv forschender Erziehungswissenschaft (z. B. Wernet 2009). Sie folgt dem Prinzip einer «extensiven Feinanalyse» (ebd., 32), nach dem nur verhältnismässig kleine Datenmengen in die Analyse einbezogen werden, diese aber äusserst genau. Die Methode geht in ihren methodologischen Prämissen davon aus, dass die in der Tiefeninterpretation anvisierten Strukturierungsprinzipien der sozialen Welt schon in kleinen Ausschnitten zur Geltung kommen und dass sie sich an anderer Stelle des Datenmaterials reproduzieren (ebd.). Im Zuge dieses zweiten Schritts greift die Analyse auf die Ergebnisse des vorhergehenden App Walk Through als Kontextwissen zurück, um beide Schritte systematisch und methodologisch konsequent miteinander zu kombinieren ${ }^{9}$. Für die in der Analyse obligatorische Fallbestimmung steht dabei die Frage im Mittelpunkt, wie sich der Student zu dem bereitgestellten Möglichkeitsrahmen der App mit seinen vielfältigen Überwachungsszenarien positioniert. Für diese Feinanalyse greifen wir diejenigen Passagen des Interviews heraus, in denen der Student unmittelbar seine Erfahrungen mit der App selbst beschreibt, da sich die fokussierten Positionierungen zwar nicht nur an ihnen, aber an ihnen eben besonders gut rekonstruieren lassen. Abschliessend kann so im Fazit vor dem Hintergrund allgemeiner Prinzipien eine empirisch begründete Fallstrukturhypothese hinsichtlich der vorliegenden Optimierungspraktiken zwischen Kontrolle und Selbstermächtigung für den individuellen Hybrid-Akteur aufgestellt werden (erste Überlegungen zu dieser Kombination von App Walk Through und Objektiver Hermeneutik finden sich bereits bei Dehmel und Burgfeld-Meise 2020, 41-42).

\section{Analyse}

\subsection{App Walk Through «Time Reduction»}

Die App Time Reduction stammt von einer kommerziellen aussereuropäischen Entwicklungsfirma, wird im Google Playstore allerdings auch in deutscher Sprache beworben. Der Werbetext propagiert sie als Vehikel, um die eigene «Smartphone-Sucht zu überwinden ${ }^{10}$. Potentielle Userinnen und User werden hier folglich als mangel-

8 Während die Objektive Hermeneutik in anderen erziehungswissenschaftlichen Subdisziplinen schon lange Verwendung findet, berücksichtigt die medienpädagogische Forschung sie bislang höchstens am Rande. Wir hoffen also auch, mithilfe dieses Beitrags die grossen Potentiale der Methode für die Medienpädagogik aufzuzeigen. Sie ermöglicht den Zugang zu aktuellen (Medien-)Phänomenen über kleine Fallzahlen, die aber dafür intensiven Analysen unterzogen werden, um so tiefe Einblicke in die Hervorbringung von Einzelfällen vor dem Hintergrund allgemeiner sozialer Regelsysteme zu erlangen.

9 Obwohl die Objektive Hermeneutik den Einbezug des Kontextwissens in der Interpretation systematisch nachlagert, hat es für die verstehende Analyse eine zentral wichtige Bedeutung und bildet eine absolut notwendige Voraussetzung für eine gelingende Fallinterpretation (siehe zur Bedeutung des Kontextwissens Wernet 2009, Kap. 3).

10 Zitate sind sinngemäss wiedergegeben. 
hafte Wesen adressiert, denen ein schwerwiegendes psychisches Problem als Nutzungsmotiv unterstellt wird. Es geht hier nicht bloss um die Optimierung des Verhaltens der Individuen mithilfe der App, sondern um deren Rettung aus einer ernsthaften Krisensituation, in deren Dienst sich die App stellt. Die App-Beschreibung suggeriert also eine Fürsorgeorientierung im Sinne der Nutzenden, für die ein hohes Mass an medizinisch-psychologischer Expertise notwendig wäre. Der Beschreibungstext startet mit der Frage, ob man das auch kenne, dass man ab und zu das Gefühl habe, zu lange und zu oft auf sein Smartphone zu starren und durch das Smartphone von anderen Dingen abgelenkt zu werden. Ein solches Gefühl hätte wohl so ziemlich jede und jeder schon einmal erlebt. Potentiell werden durch die App also alle Besitzerinnen und Besitzer eines Smartphones angesprochen und als süchtig klassifiziert. Die Smartphone-Nutzung wird hier pauschal pathologisiert, das Herunterladen der App wird zunächst zum Symbol für das Eingeständnis der eigenen Sucht.

Nach der Installation fordert die App dazu auf, die Sprache und anschliessend das angestrebte Nutzungsziel auszuwählen. Dabei geht es auf der einen Seite um die Reduktion der täglichen Nutzungszeit und auf der anderen Seite um die der Anzahl der täglichen Entsperrungen des Geräts, die durch die App protokolliert werden. Die App macht den Vorschlag von $1 \mathrm{~h} 30$ min täglicher Nutzung und 50 täglichen Entsperrungen und suggeriert somit Expertise hinsichtlich guter und schlechter Werte. Woher diese stammen und auf welcher Grundlage sie getroffen werden, wird nicht transparent. Die Werte sind über Schieberegler beliebig und unabhängig voneinander veränderbar, letztlich werden somit die Userinnen und User von der App in die Pflicht genommen, Verantwortung über ihre eigene Smartphone-Routinen zu übernehmen. Die App stellt nur die Möglichkeit zur Selbstkontrolle bereit, die Festlegung und das Erreichen des angestrebten Optimierungsziels hinsichtlich der Reduktion der Gerätenutzung muss allerdings durch die Userinnen und User selbst erreicht werden.

Im nächsten Schritt fordert die App dazu auf, die Datenschutzbestimmungen zu akzeptieren. Diese Aufforderung liest sich wie eine Art Eingeständnis eines unangenehmen Erfordernisses, um bei der Überwindung der zugeschriebenen Sucht unterstützen zu können. Die App suggeriert so eine therapeutische Notwendigkeit dieser Bestimmungen für die Gesundheit der Nutzenden. Sie verweist zudem darauf, man solle sich keine Gedanken hinsichtlich des Datenschutzes machen, da die Nutzungsdaten nur auf dem Smartphone gespeichert werden würden. Schaut man sich die Bestimmungen aber näher an, fällt auf, dass diese nur in englischer Sprache einsehbar sind. Userinnen und User müssen der App erlauben, auf die Logfiles des Gerätes zuzugreifen, sodass die App das gesamte Smartphone-Nutzungsverhalten also nicht nur der App Time Reduction - detailliert nachvollziehen kann. Sie werden laut den Bestimmungen für die Optimierung der App-Angebote des Herstellers sowie zur Schaltung von individualisierter Werbung verarbeitet. Letztlich wird von der App mit dem Versprechen, dass die Nutzungsdaten nur auf dem Smartphone gespeichert 
werden, falsche Assoziationen geschaffen. Die Daten werden sehr wohl für kommerzielle Zwecke und somit im ökonomischen Interesse des Herstellers verwendet. Stimmt man diesen Datenschutzbestimmungen nicht zu, kann die App nicht genutzt werden. Die Userinnen und User müssen also die umfassende Kontrolle durch den App-Hersteller erlauben. Zudem kann eine kostenpflichtige Version der App mit zusätzlichen Extrafunktionen und ohne geschaltete Werbung gekauft werden.

Nach der Zustimmung zu den Datenschutzbestimmungen öffnet sich der Startbildschirm der App. Nutzende werden mit einer schier unüberschaubaren Flut von Informationen überhäuft. An vorderster Stelle findet sich ein grosses Kreisdiagramm, auf dem die - gemessen an den eingestellten Zielen - noch verbleibende Nutzungszeit und die noch übrigen Entsperrungen angezeigt sind. Wenn dieses Diagramm angeklickt wird, entfaltet sich ein Zeitstrahl des aktuellen Tages, auf dem detailliert aufgelistet ist, zu welchen Zeiten welche App wie lange verwendet wurde. In den Stunden, in denen das Smartphone gar nicht genutzt wurde, wird ein glücklicher Smiley abgebildet. Mit einer Wischbewegung nach rechts zeigt die App die heutigen Nutzungszeiten der installierten Apps über einen Balken, der bei ihrer Verwendung ansteigt. Befindet sich die Nutzungszeit einer App unterhalb von 30 Minuten, ist dieser grün, steigt sie darüber hinaus, setzt sich der Balken in roter Farbe fort. Zurück auf dem Startbildschirm finden sich unter dem Kreisdiagramm die beiden innerhalb der letzten sieben Tage meistgenutzten Apps und das von der App eingeschätzte Suchtlevel. Die App unterscheidet hier zwischen fünf Abhängigkeitsstufen, die Eingruppierung erfolgt unabhängig von den gesetzten Zielen. Daneben finden sich Beschreibungen der unterschiedlichen Stufen, diese werden rein quantitativ anhand der durchschnittlichen Gesamtnutzungszeit des Smartphones innerhalb der letzten Woche festgelegt. Auffällig ist, dass auch innerhalb des obersten Niveaus, mit einer durchschnittlichen Nutzungszeit von unter einer Stunde täglich, davor gewarnt wird, man könne ständig «rückfällig» werden, sodass ein Bedrohungsszenario entsteht, das es in der Therapielogik der App sinnvoll erscheinen lässt, diese nicht zu löschen.

Darunter folgen Angaben zur Nutzung von «produktiven Apps» mit einer Minutenangabe des heutigen Tages. Diese können nur in der kostenpflichtigen Version genauer eingesehen werden. Zudem wird die Verwendung dieser «produktiven Apps» nicht von der Gesamtzeit abgezogen. Dann werden zwei Balkendiagramme zu den Werten des heutigen Tages in Halbstundenintervallen und zur letzten Woche (jeweils getrennt nach Zeit und Bildschirmentsperrungen) gezeigt. Auffällig ist, dass die App direkt nach der Installation auch Werte anzeigt, auf die sie noch gar nicht auf dem Gerät zugreifen konnte. Darunter können die Userinnen und User Zugang zu ihrem wöchentlichen und monatlichen Gesamtbericht bekommen, dafür muss allerdings entweder ein Werbevideo angeschaut oder aber die kostenpflichtige Version gekauft werden. Zu guter Letzt finden sich sogenannte «Herausforderungen», die jeweils darin bestehen, eine bestimmte App nur für einen bestimmten Zeitraum nicht oder nur 
eingeschränkt zu nutzen, oder aber für einige Stunden gänzlich auf das Smartphone zu verzichten. Gamification spielt hier also auch eine gewisse Rolle, ist allerdings nicht der Hauptfokus der App. Es wird im Grunde eine paradoxe Situation geschaffen, indem durch Gamification und die suggerierte Bedeutung der App zur Suchtbekämpfung die Beschäftigung mit der App verstärkt wird, obwohl die Nutzungszeiten des Smartphones reduziert werden sollen.

Das App-Interface wird von verschiedenen Buttons gerahmt. Am oberen Ende befindet sich ein Frame mit einer Schaltfläche zu den Einstellungen und zu einem Warenkorb, über den die kostenpflichtige Version erworben werden kann. Insgesamt ist auffällig, an wie vielen Stellen die App Nutzende zu dieser Bezahlversion weiterleitet. Der Kauf der Proversion wird dabei immer wieder als Notwendigkeit im Sinne der Suchtüberwindung beworben, um noch detailliertere Einschätzungen hinsichtlich der eigenen Gerätenutzung zu erlangen. Am unteren Rand des App-Interface ist zunächst eine Leiste, über die Werbung angezeigt wird, in unserer Analyse interessanterweise auch für Smartphone-Apps, was dem vermeintlich therapeutischen Ziel der App entgegensteht. Darunter findet sich ein Frame mit einer Schaltfläche, über die neue «Herausforderungen» (s.o.) gestartet werden können, eine zum Home Bereich der App, ein Link zu den «Tagesauswertungen» und zum «Nutzerprofil». In der Schaltfläche «Tagesauswertungen» findet sich eine Tagesübersicht mit den Werten hinsichtlich der Nutzungszeit und zu den Entsperrungswerten, die jeweils mit dem Vortag verglichen werden. Sind sie angestiegen, wird der prozentuale Anstieg rot hinterlegt, sind sie gesunken, erscheinen sie grün. In der Tagesansicht wird der detaillierte Zeitstrahl mit den jeweils genutzten Apps angezeigt. Durch die Öffnung einer anderen App wird am Rand des Screens durch eine Uhr die tagesaktuelle Nutzungsdauer eingeblendet. Diese ist zu Beginn grün hinterlegt, steigt die Nutzungszeit über zwanzig Minuten erscheint sie gelb und bei einem Wert von über 30 Minuten wird sie rot; zusätzlich erscheint ein Warnhinweis, dass die App möglichst heute nicht mehr genutzt werden sollte - unabhängig davon, um welche App es sich handelt. Dieser Warnhinweis lässt sich mit einem Klick auf einen Button mit der Aufschrift «nur noch 2 Minuten» oder «ermahne mich heute nicht nochmal» wegklicken. Beide Optionen erinnern an ein Erziehungsgespräch zwischen Eltern und Kindern, in dem etwa um die Fernsehzeit gerungen wird. Die App übernimmt hier die Rolle der oder des mahnenden Erziehungsberechtigten, die Userinnen und User begeben sich in die Rolle des oder der zu Erziehenden. Diese Funktionen können in den Einstellungen allerdings ausgeschaltet werden.

Der im Austausch mit den menschlichen Akteuren abgesteckte Möglichkeitsrahmen der App schreibt Userinnen und Usern eine fast gänzlich passive Rolle zu, in der sie zu Empfängerinnen und Empfängern von mahnenden Disziplinierungen werden, gegen die sie sich innerhalb der App nicht «zur Wehr» setzen können (nur durch das Löschen der App). Die Protokollierung des Verhaltens wird im vorliegenden Fall 
ausschliesslich durch die App vorgenommen. Lediglich bei den Herausforderungen kann aktiv ausgewählt werden, allerdings werden von der App hier Vorschläge unterbreitet. Sie bewertet die Smartphone-Nutzung pauschal als schlecht (im Übrigen auch die Nutzung von Time Reduction selbst) und als Zeitverschwendung. Sie wird also unabhängig von der inhaltlichen Fokussierung pathologisiert. Das Nutzungsverhalten wird in diesem Zusammenhang drastisch reflektiert, die App stellt ausschliesslich anhand der durchschnittlichen Verwendungszeit des Smartphones eine schwerwiegende Diagnose als «süchtig» und bewertet über farbliche Darstellung und Warnhinweise die Nutzung, ohne dass transparent wird, auf Basis welcher Expertise dies eigentlich geschieht. Der Möglichkeitsrahmen der App konstituiert somit ein erzieherisches Machtungleichgewicht. Nutzende müssen diesen Werten blind vertrauen, ohne sie in irgendeiner Weise überprüfen zu können. Sie sind somit auf die Beobachtung des eigenen Selbst und das Empfangen von Anweisungen verwiesen, der App kommt die Funktion einer (vermeintlich) objektiven und unbestechlichen Kontrollinstanz zu, die den Selbstoptimierungsprozess begleitet.

Es kann eine schier unüberschaubare Anzahl von Statistiken eingesehen werden. Den Userinnen und Usern wird so der Eindruck vollumfänglicher Überwachung suggeriert, der sie nicht entkommen können. Nur durch eine tatsächliche Veränderung des Verhaltens (in der Optimierungslogik der App die Reduktion der SmartphoneNutzung) können die in der App reflektierten Werte verbessert werden. Insofern lässt sie sich als prototypisches Beispiel eines Panopticons im Sinne Foucaults im Kontext der Verdatung deuten. So wird aus einem diffusen Gefühl, das Smartphone zu oft in der Hand zu halten, eine vermeintlich objektive in Zahlen manifestierte Gewissheit (Subjektwissen), ein schwerwiegendes Problem zu haben.

Das in der Optimierungslogik der App zu erreichende Verhaltensoptimum würde in einem gänzlichen Verzicht liegen. Innerhalb des Möglichkeitsrahmens der App wird somit ein Paradox zwischen der ökonomischen und der (vermeintlichen) therapeutischen Zielsetzung deutlich. Der App-Hersteller verzeichnet dann den meisten Gewinn, wenn möglichst viele Userinnen und User die App herunterladen und ihr Smartphone möglichst oft und lange nutzen, da somit am meisten Nutzungsdaten gesammelt und möglichst viel Werbung geschaltet werden kann. Gleichzeitig verschreibt sich die App auf der inhaltlichen Ebene aber der Reduktion der Smartphone-Nutzung, im Optimalfall dieser Logik macht sie sich sogar überflüssig. Gamification-Elemente und die vielfach beschworene Rückfallgefahr in die Sucht sollen dies freilich verhindern. Beide Zielsetzungen stehen sich diametral entgegen und sind nicht miteinander vereinbar. Dem begegnet der Hersteller mit der innerhalb des Möglichkeitsrahmens konstruierten Expertinnenrolle omnipräsenten Empfehlung, die Proversion zu kaufen und so ebenfalls Gewinne zu erzielen. Zudem wird durch die kostenpflichtige Version weiteres Optimierungspotential suggeriert. Es gibt in dieser Logik ein Mehr an Daten, konkrete Statistiken, ein genaueres Nachvollziehen, Bekämpfen und Kontrolle über die eigenen (vermeintlichen) Suchtmechanismen. 


\subsection{Interviewanalyse mit der Objektiven Hermeneutik}

$\mathrm{S}$ : besonders aufgefallen is mir dass ähm, ich halt wirklich das Smartphone ähm . ja sehr exzessiv benutze, ähm da kommen teilweise Stunden zusammen das hätte ich mir gar nicht vorstellen können ${ }^{11}$

In den ersten Sequenzen deutet der Student die gemachten Erfahrungen im Austausch mit der App als Intervention hinsichtlich seiner eigenen Smartphone-Nutzung. Es geht hier nicht um eine qualitative Ergründung der Nutzungsweisen und deren Einordnung, sondern um die quantitative Erfassung der Zeit. Auf der latenten Sinnebene schafft diese Ergründung im Interviewtext eine Objektivierung eines vorher diffusen Gefühls hinsichtlich der täglichen Bildschirmzeit, die durch die Vermessung auf konkrete Zahlen gebracht wird. Der Student übernimmt folglich die durch die App vorgegebenen Implikationen als neues Subjektwissen. In diesem Zusammenhang verweist er auf einen Erkenntnisprozess, dass sich die täglichen Routinen oberhalb eines spezifischen Grenzwertes befinden müssen und dass die zuvor lediglich grob mögliche subjektive Einschätzung sinnlogisch deutlich niedriger gelegen haben muss.

Sein Nutzugsverhalten reflektiert er im Anschluss an die von aussen an ihn herangetragenen Werte als «exzessiv», ein Begriff den man im üblichen Sprachgebrauch im Zusammenhang mit Suchtmittelmissbrauch verorten könnte. Unter Einbezug des Kontextwissens aus dem Walk Through wird hier also ersichtlich, dass der Student an dieser Stelle des Interviews die in die App eingeschriebene Suchtrhetorik übernimmt. Dabei ist augenfällig, dass er im Zusammenhang mit dieser Einschätzung im Präsens und nicht in der Vergangenheit spricht («benutze»). Sinnlogisch muss es sich also um einen noch anhaltenden Zustand handeln, der zum Zeitpunkt des Sprechens nicht oder noch nicht überwunden worden ist, oder aber als nicht-krisenbehaftet und somit als wenig bedeutsam bewertet wird.

S: andererseits begründe ich das ganze irgendwie dann damit dass ich das Handy wirklich für alles nutze äh sei es für irgendwelche Zahlungen, ähm Kommunikation natürlich über WhatsApp, äh Sachen nachschlagen im Internet, informieren darüber was es in der Mensa gibt und ähm, auch so ganz banale Sachen wie die Uhrzeit checken ich hab halt keine Uhr und deswegen guck ich halt auf mein Smartphone, aber ich glaub sehr viel Zeit geht auch einfach, ins Smartphone weil man sich die Zeit vertreiben will, ähm, und auch weil man Medien konsumieren will, seis nun Musikhören oder Videosgucken oder Filmgucken, vieles macht man tatsächlich mittem Smartphone

11 Symbolbedeutungen: , = kurzes Absetzen im Sprechen; . = ca. 1 Sek. Pause; (2) = ca. 2 Sek. Pause; sch- = im Sprechen abgebrochenes Wort. 
Im Anschluss folgt eine Rechtfertigung. Der Student arbeitet sich somit an seinem offenbar starken Abweichen von der gesetzten Norm hinsichtlich angemessener Smartphone-Nutzungswerte ab und nennt anschliessend unterschiedliche Beispiele seiner Nutzungsweisen, die eine vollumfängliche Integration des Gerätes in den Alltag dokumentieren. Die aufgeführten Nutzungsbeispiele lassen sich in die von Möller (2016) herausgearbeiteten Kategorien zur Bedeutung von Smartphones im Leben von Studierenden einordnen: Es wird im vorliegenden Fall zum personalisierten Verwaltungs- und Informationszentrum und zum Medium der sozialen Eingebundenheit (ebd., 191-98). Das Smartphone ist folglich ein integraler Bestandteil der alltäglichen Lebensbewältigung, womit die hohen Nutzungswerte im Interview gerechtfertigt werden.

Gleichzeitig perspektiviert der Student das Gerät aber auch unter dem Aspekt der Unterhaltung und des Zeitvertreibs. Interessanterweise wechselt er hier den Sprechmodus und bezieht dies nicht mehr auf seine eigene Person, sondern formuliert eine allgemeine Regel («weil man sich die Zeit vertreiben will») vor dem Hintergrund seiner Erfahrungswelt. Innerhalb des sozialen Bezugssystems erscheint die von ihm an den Tag gelegte Nutzungsweise also üblich zu sein. Während die ersten Punkte ein aktives Handeln im Austausch mit dem Gerät notwendig machen, handelt es sich der Argumentationslogik des Studenten nach bei den zweiten um sogenannte passive Nutzungsroutinen, die auf reines, von ihm so bezeichnetes «Konsumieren» beschränkt sind. Er nimmt hier also eine feine Differenzierung der von den Zeitnormen abweichenden Smartphone-Nutzung vor und hält sie den von der App Time Reduction eruierten rein quantitativen Werten entgegen.

S: ja, hmm und was mir sonst noch aufgefallen ist, ähm, die App schreibt einem, oder die App gibt äh, die ich jetzt genutzt hab, schreibt einem vor , oder lässt einen entscheiden, ähm, wie viel wie die maximale ähm Nutzungsdauer sein soll also das man sich quasi selber Ziele setzen kann, und wie oft man sein Handy unlocked oder halt entsperrt . und ähm ja das kann man halt selber festlegen aber es gibt auch irgendwie ne Skala die besagt ab sieben Stunden sch- Handynutzung am Tag . ähm . is man halt schon, süchtig komplett süchtig, da gibts sonne Skala und äh, ja da würd mich halt interessieren ob auf was für wissenschaftlichen Theorien oder Studien oder was auch immer, diese Skala eigentlich beruht oder ob die sich das einfach aus den Fingern gesogen haben

Der zuvor benannte Punkt zur Einordnung der Nutzungswerte scheint nun abgeschlossen zu sein und der Student wendet sich einem inhaltlichen Punkt zu, der ihm "sonst noch aufgefallen ist». Hier geht er auf die innerhalb des Möglichkeitsrahmens der App eingeschriebenen Handlungsalternativen ein, sich individuelle Nutzungsziele zu setzen. Auf der latenten Sinnebene wird an dieser Stelle die innerhalb des Walk 
Throughs interpretierte weitgehend passive Rolle deutlich, die Userinnen und Usern innerhalb des Hybrid-Akteurs im Austausch mit der App einen nur sehr geringen Spielraum hinsichtlich ihrer aktiven Handlungsmöglichkeiten lässt. Die App «lässt einen entscheiden», welche Ziele man sich setzt; es ist also eine Erlaubnis notwendig und die Handlungen unterliegen der Kontrolle und der Steuerung der App.

Im Anschluss geht der Student auf die von der App vorgenommenen Diagnose eines Suchtniveaus ein. Zunächst wird eine gewisse Überraschung darüber deutlich, ab einem Wert von sieben Stunden täglicher Nutzung «schon komplett süchtig» zu sein. Dass eine solche Abstufung einer Sucht durch den Möglichkeitsrahmen der App rein anhand der Nutzungszeit überhaupt vorgenommen wird, bewertet er auf der latenten Sinnebene des Interviews damit als legitim, stellt allerdings die Skalenbreite in ihrer Wissenschaftlichkeit in Frage, die bestimmten Werten ein spezifisches Suchtlevel zuteilt. Der Student reflektiert somit die in der App konstruierte vermeintlich wissenschaftliche Expertise der App zur «Behandlung» der Smartphone-Sucht und zweifelt ihren Status einer wirklich objektiven Kontrollinstanz an. Er ordnet den Wert von sieben Stunden an dieser Stelle vor dem Hintergrund seiner subjektiven Erfahrungswerte ein, auf der latenten Sinnebene des Interviews bewertet er die von der Skala vorgenommene Zuteilung als zu streng. Diese Einordnung dokumentiert erneut die vollumfängliche Integration des Smartphones in den Alltag als gängige Norm innerhalb der Erfahrungswelt des Studenten.

Zum Schluss beziehen wir noch eine Interviewpassage mit in die Interpretation ein, in der der Student seine Erfahrungen mit der App am Ende des Interviews nochmals abschliessend zusammenfasst.

S: (2) ähm . ja wie gesagt wie schon gesagt die Erkenntnis die war halt, ähm, schon irgendwie augenöffnend und äh, war auf jeden Fall interessant darüber mal aufgeklärt zu werden wie viel Zeit man in dieses Gerät investiert, aber ich hab jetzt nicht irgendwie die Erfahrung gemacht dass ich dadurch aufgrund dieser Erkenntnis ähm den Drang dazu hab äh , mein Konsumverhalten zu ändern

In dieser abschliessenden kurzen Beschreibung seiner Erfahrungen reflektiert er diese als die Gewinnung einer «Erkenntnis», die für ihn «augenöffnend» war und ihn «aufgeklärt» hat. Er nutzt an dieser Stelle also drei Begriffe, die auf eine potentiell lebensverändernde Bedeutung der mit der App gesammelten Erfahrungen hinweisen und sich in die zuvor interpretierte Übernahme der Suchtrhetorik eingliedern. Dieser für ihn vorher nicht ersichtliche Zusammenhang seines alltäglichen Lebens wird überhaupt erst durch den Austausch mit der App zu einem sichtbaren Umstand, erst durch die Überwachung der App erscheint inm überhaupt die Zuschreibung als «Smartphone-süchtig» als legitim. Aus einer bildungstheoretischen Perspektive lassen diese beschriebenen Erfahrungen das Potential einer Krisenerfahrung deutlich 
werden, denen die Entwicklungsoption einer Veränderung der Selbst-/Weltverhältnisse als Bildungserfahrung (Koller 2010) innewohnt. Diese Sprechweise des Studenten übernimmt somit die in die App eingeschriebene Therapielogik, die über ein Aufzeigen der Nutzungszeit eine Verhaltensmodifikation auf Seiten der Userinnen und User anstrebt und somit potentiell selbstermächtigend hinsichtlich neuer Freiheiten in der Alltagsgestaltung jenseits des Smartphones wirken kann. Solches findet auch in dem von ihm genutzten Begriff «Konsumverhalten» Ausdruck, der sich ebenso in den Sucht-/Therapiekontext eingliedert.

Letztlich distanziert er sich allerdings von diesen Nutzungswerten, die im Möglichkeitsrahmen der App suggerierte vollumfängliche Überwachung (Panoptismus) wird durch den Studenten somit relativiert. Die Werte mögen zwar hoch sein, er sieht allerdings keinerlei Bedarf, sie zu verändern, da der mit ihnen einhergehende Nutzen (er spricht von einer «Zeitinvestition» ins Smartphone) offenbar zu gross ist. Auf der latenten Sinnebene wird also anhand des generierten Subjektwissens eine bewusste und reflexive Entscheidung deutlich, trotz der generierten «augenöffnenden» Werte, die Smartphone-Routinen beizubehalten. Er widersetzt sich somit bewusst den von dem Möglichkeitsrahmen der App geschaffenen normativen Setzungen hinsichtlich des angestrebten Optimierungshandelns, denen er die suggerierte objektive Gültigkeit abspricht. Er bricht also das gemeinsame Handeln innerhalb des Hybrid-Akteurs auf. Dass in den vom Studenten aber durchaus akzeptierten Therapiezusammenhang der App ökonomische Interessen des Herstellers eingewoben sind, dass es also innerhalb des bereitgestellten Optimierungsszenarios schwerpunktmässig um das Verdienen von Geld und nicht um die Hilfe zur Überwindung einer Sucht geht und dass die vermeintliche Therapielogik lediglich das dafür eingesetzte Vehikel ist, wird im Interview nicht reflektiert. Die damit einhergehende Überwachung des gesamten Smartphone-Verhaltens geschieht im Verborgenen und wird für Userinnen und User in der App-Nutzung nur in der geschalteten Werbung transparent. Interessant ist also, dass die paradoxen Konstruktionen der App auch in der Aneignung und InBeziehung-Setzen des Studenten aufscheinen, wenngleich beide Seiten die monetäre Gewinnerzielungsabsicht durch den Verkauf von Nutzungsdaten konsequent ausblenden.

\section{Fazit}

Aus den theoretischen Rahmungen und empirischen Interpretationen lassen sich nun zentrale Perspektiven für Optimierungen zwischen Kontrolle und Ermächtigung für den Hybrid-Akteur insgesamt ableiten. Übergreifend lässt sich durch die empirische Analyse im Lichte der Theorien von Foucault und Deleuze konstatieren, dass ein gesellschaftlicher Optimierungsimperativ durchaus wirkmächtig ist und sich in solchen Self-Tracking-Apps besonders anschaulich manifestiert. Dennoch sind einfache 
Dualismen von Disziplinierung und Kontrolle versus Ermächtigung nicht zutreffend. Sie können auf unterschiedlichen Ebenen durchaus anders ausgeprägt sein. Zunächst einmal sammelt das Individuum (zugleich auch unbemerkt der App-Hersteller und einbezogene Dritte) Daten über das eigene Verhalten. Langfristig gesehen kann so ein grosses Datenvolumen an Wissen über eigene Nutzungsweisen angelegt werden, die auch entgegen der App interpretiert werden können. Das eingangs fokussierte Subjektwissen liesse sich somit auf drei unterschiedlichen Ebenen verorten: Das Experiment des Studenten liefert Hinweise darauf, dass der Expertinnenlogik der App nicht gänzlich gefolgt wird und dementsprechend eigene Interpretations- und Legitimierungsansprüche (sozusagen eigenes Subjektwisssen) wirksam werden. Die mit Bezug zu Becks Risikogesellschaft dargelegte potentielle Orientierungsfunktion von Self-Tracking-Apps wird im vorliegenden Fall also nicht in den Alltag übernommen. Dies ist durchaus als Ermächtigungsperspektive zu betrachten, die sich im InBeziehung-Setzen als Hybrid-Akteur für den Studenten als intentional Handelnden vollzieht. Der Student als User behält hier innerhalb der gemeinsamen Praxis des Hybrid-Akteurs die Oberhand. Übergreifend ist es durch solche individuellen Datensammlungen und deren Interpretation, die auch durchaus ausserhalb der SelfTracking-App-Anbieter-Logik liegen kann, möglich, eine ganz andere Wissensbasis für medizinische, therapeutische, soziale etc. Interventionen zu schaffen. Neben der etablierten Studienlage durch Daten von Probandinnen und Probanden, Kohorten und Stichproben entsteht eine Datenerhebung auf individueller Basis (wobei die Qualität der erhobenen Daten sicherlich variieren kann). Sie kann die zuvor genannten professionellen Interventionen durchaus bereichern, indem mehr Wissen über das konkrete Subjekt in die Bewertung einfliesst und sich so in spezifischen Selbst-/ Weltverhältnissen manifestiert, was als empirisches Subjektwissen umschrieben werden könnte (Burgfeld-Meise 2020).

Was im vorliegenden Fall ein Defizit bleibt, ist die Reflexion der Datensammlung über das Subjekt durch die App- und Smartphone-Hersteller sowie mit diesen kooperierenden Dritten, als weitere Ebene des Subjektwissens (als Datensammlung über das Subjekt). Es werden unsichtbar Informationen zusammengetragen, deren Interpretation und weitere Verwendung im schlechtesten Fall, wie durch Deleuze skizziert, die Freiheits- und Gleichheitsrechte des Einzelnen empfindlich beschränken können. In Analogie zu Foucaults Panoptikum ist der Überwachungsturm der Datensammlung unsichtbar hinter der Überwachung des Subjekts durch das App-Interface und daher nur selten Thema der subjektiven Reflexionen - wie es auch die vorliegende Fallinterpretation zeigt. Hier bedarf es weiterer Sensibilisierungsangebote und alternativer Handlungsempfehlungen, damit das Subjekt sich informiert und reflektiert zu diesen Datensammlungs- und Verwertungskontexten ähnlich versiert wie auf der offensichtlicheren inhaltlichen Ebene positionieren kann. 


\section{Literatur}

Beck, Ulrich. 1986. Risikogesellschaft: Auf dem Weg in eine andere Moderne. Frankfurt a. M.: Suhrkamp.

Bettinger, Patrick. 2017. «Hybride Subjektivitäten in mediatisierten Welten als Bezugspunkte der erziehungswissenschaftlichen Medienforschung». MerzWissenschaft 61(6): 7-17.

Bublitz, Hannelore. 2014. «Subjekt». In Foucault-Handbuch: Leben - Werk - Wirkung, hrsg. v. Clemens Kammler, Rolf Parr, und Ulrich J. Schneider, 293-96. Stuttgart: J.B. Metzler. https://doi.org/10.1007/978-3-476-01378-1_44.

Burgfeld-Meise, Bianca. 2020. «(Medien-)Bildungskulturen im Wandel: Digitalisierung als Katalysator veränderter Wissenzirkulationen». Zeitschrift MedienPädagogik 37: 67-83. https:// doi.org/10.21240/mpaed/37/2020.07.04.X.

Dehmel, Lukas, und Bianca Burgfeld-Meise. 2020. «Vergissmeinnicht! Self-Tracking-Apps auf dem Smartphone als Erinnerungsräume junger Erwachsener». MerzWissenschaft 64(6): 3847.

Dehmel, Lukas, und Sebastian Zick. 2020. «Das Smartphone in der berufsvorbereitenden Bildung junger Erwachsener: Empirische Erkundungen aus praxistheoretischer Perspektive». In Praxistheoretische Perspektiven in der Medienpädagogik, hrsg. v. Patrick Bettinger, und Kai-Uwe Hugger, 179-96, Wiesbaden: Springer VS. https://doi.org/10.1007/978-3-65828171-7_10.

Deleuze, Gilles. 1993. «Postskriptum über die Kontrollgesellschaften». In Unterhandlungen. 1972-1990, hrsg. v. Gilles Deleuze, 254-62. Frankfurt a. M.: Suhrkamp.

Foucault, Michel. 1976. Überwachen und Strafen: Die Geburt des Gefängnisses. Frankfurt a. M.: Suhrkamp.

Gerhardts, Lara, Lukas Dehmel, und Dorothee M. Meister. 2021. «Die berufliche Tabletaneignung von Lehrkräften als Beispiel der Mediatisierung pädagogischer Handlungskontexte: Theoretische Perspektiven und empirische Befunde». In Jahrbuch Medienpädagogik 16: Medienpädagogik in Zeiten einer tiefgreifenden Mediatisierung, hrsg. v. Karsten D. Wolf, Klaus Rummler, Patrick Bettinger, und Sandra Aßmann, 129-159. https://doi.org/10.21240/ $\mathrm{mpaed} / \mathrm{jb} 16 / 2021.01 .15 . \mathrm{X}$.

Hummrich, Merle. 2016. «Was ist der Fall? Zur Kasuistik in der Erziehungswissenschaft». In Was ist der Fall? Kasuistik und das Verstehen pädagogischen Handelns, hrsg. v. Merle Hummrich, Astrid Hebenstreit, Merle Hinrichsen, und Michael Meier, 13-38. Wiesbaden: Springer VS. https://doi.org/10.1007/978-3-658-04340-7_2.

Keuneke, Susanne. 2005. "Qualitatives Interview». In Qualitative Medienforschung: Ein Handbuch, hrsg. v. Lothar Mikos, und Claudia Wegener, 254-67. Konstanz: UKV.

Koller, Hans-Christoph. 2010. «Grundzüge einer Theorie transformatorischer Bildungsprozesse». In Gesellschaftliche Bedingungen von Bildung und Erziehung: Eine Einführung, hrsg. v. Andrea Liesner, 288-300. Stuttgart: Kohlhammer. 
Latour, Bruno. 2006. «Über technische Vermittlung: Philosophie, Soziologie und Genealogie». In ANTology: Ein einführendes Handbuch zur Akteur-Netzwerk-Theorie, hrsg. v. Adréa Belliger, und David J. Krieger, 483-528. Bielefeld: Transcript.

Light, Ben, Jean Burgess, und Stefanie Duguay. 2018. «The walkthrough method: An approach to the study of apps». New Media \& Society 20(3): 881-900. https://doi. org/10.1177/1461444816675438.

Möller, Renate. 2016. «Das Smartphone als Leitmedium». In Ent-Grenztes Heranwachsen, hrsg. v. Ulrike Becker, Henrike Friedrichs, Friederike von Gross, und Sabine Kaiser, 185-99. Wiesbaden: Springer VS. https://doi.org/10.1007/978-3-658-09793-6_10.

Nohl, Arnd-Michael. 2011. Pädagogik der Dinge. Bad Heilbrunn: Verlag Julius Klinkhardt.

Rammert, Werner, und Ingo Schulz-Schaeffer. 2002. «Technik und Handeln: Wenn soziales Handeln sich auf menschliches Verhalten und technische Abläufe aufteilt». In Können Maschinen handeln? Soziologische Beiträge zum Verhältnis von Mensch und Technik, hrsg. v. Werner Rammert, und Ingo Schulz-Schaeffer, 11-64, Frankfurt a.M.: Campus.

Selke, Stefan. 2016. «Ausweitung der Kampfzone: Rationale Diskriminierung durch Lifelogging und die neue Taxonomie des Sozialen». In Lifelogging: Digitale Selbstvermessung und Lebensprotokollierung zwischen disruptiver Technologie und kulturellem Wandel, hrsg. v. Stefan Selke, 309-39. Wiesbaden: Springer VS. https://doi.org/10.1007/978-3-658-10416-0_14.

Weich, Andreas. 2017. Selbstverdatungsmaschinen: Zur Genealogie und Medialität des Profilierungsdispositivs. Bielefeld: Transcript. https://doi.org/10.14361/9783839439425.

Wernet, Andreas. 2009. Einführung in die Interpretationstechnik der Objektiven Hermeneutik. 3. Aufl. Wiesbaden: VS Verlag. https://doi.org/10.1007/978-3-531-91729-0. 